\title{
An Effective Conversational Agent with User Modeling based on Bayesian Network
}

\author{
Seung-Ik Lee, Chul Sung, and Sung-Bae Cho \\ Computer Science Department, Yonsei University, 134 Shinchon-dong, Sudaemoon-ku, Seoul \\ 120-749, Korea \\ \{cypher, sungc\}@candy.yonsei.ac.kr, sbcho@csai.yonsei.ac.kr
}

\begin{abstract}
Conversational agents interact with users using natural language interface. Especially in Internet space, their role has been recently highlighted as a virtual representative of a web site. However, most of them use simple pattern matching techniques without considering user's goal. In this paper, we propose a conversational agent that utilizes user model constructed on Bayesian network for the responses consistent with user's goal. The agent is applied to the active guide of a website, which shows that the user modeling based on Bayesian network helps to respond to user's queries appropriately with the their goals.
\end{abstract}

\section{Introduction}

Most Internet sites provide a simple keyword-based search engine, which is difficult to give right information because they do not consider user's goals. As an alternative for the dumb interfaces of web sites, conversational agents are recently being developed because they have conversations with users by processing natural language. However, most conversational agents, e.g., Eliza [1] and ALICE(Artificial Linguistic Internet Computer Entity, http://www.alicebot.org), have the shortcomings of not being able to take into accounts user's intentions because of their simple sequential pattern matching based on keywords.

One of the techniques for modeling user's intentions or goals is to use Bayesian network. It is an intuitive and parsimonious representation of probability distributions and effective in diagnosing user's needs and thus provides useful enhancements to legacy software applications when embedded within them. Pynadath [2] used probabilistic models for making inferences about the goals of car drivers in navigation. Albrecht [3] applied Bayesian models in action prediction in a multi-user computer game. Horvitz [4] worked to build models for inference and decision-making under uncertainty about user's goals at each level of the task hierarchy with Bayesian networks.

In this paper, we propose a conversational agent that can have more intelligent conversations by inferring diverse user goals through user modeling based on Bayesian network and apply it to the guide of a website to show the usefulness.

\section{Conversational Agent}

Fig. 1 shows the overall structure of the conversational agent. After preprocessing user's queries to correct typos and supplant synonyms, Goal Inference module infers user's 


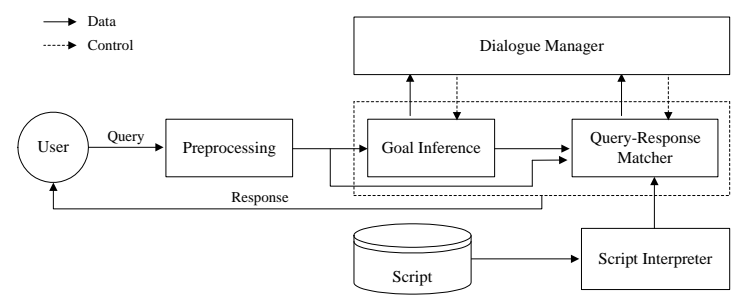

Fig. 1. The architecture of a conversational agent

goals. When this module cannot infer user's goals because of insufficient information, the Dialogue Manager requests the user to give more information. Once the goal is inferred, the agent presents an appropriate answer by searching the knowledge base or script. This requires the Goal Inference to play an important role in finding the appropriate answer by reasoning goals during the mixed-initiative interactions with the user [7].

\subsection{User Model based on Bayesian Network}

Fig. 2 shows the user model for the introduction of a web site with Bayesian network. Evidence variables of a node are represented as parent nodes. Each evidence variable represents a set of keywords. For example, the evidence variable 'place' represents keywords like 'position' and 'location'. We construct a user model for the introduction of a specific site with Bayesian network. When the information from a query is insufficient

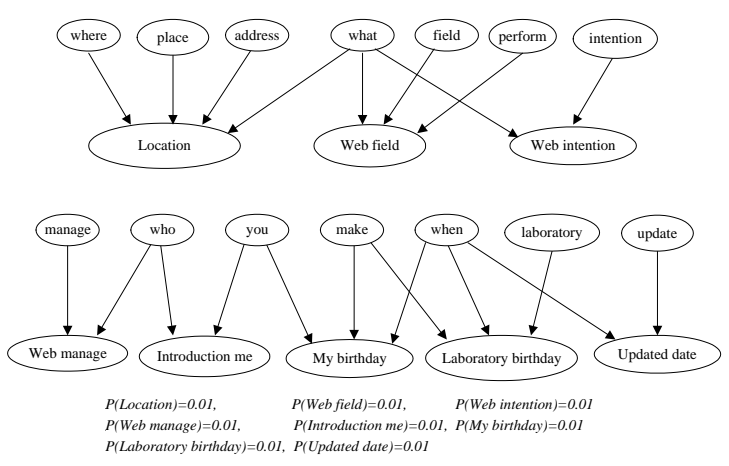

Fig. 2. Bayesian network architecture for modeling user goals

for the transition from the current goal to a more detailed one, the agent requests more information to the user. 


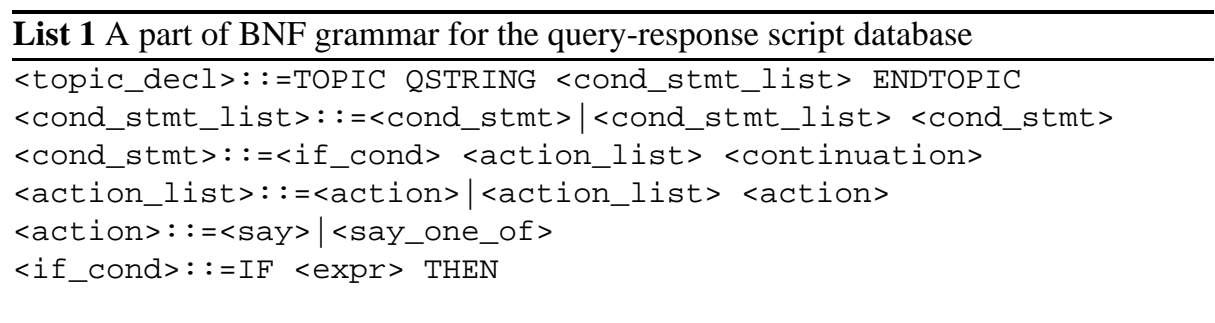

\subsection{Knowledge Representation and Matching}

Knowledges for the matching of queries to responses are described in a database called script and a part of the script grammar is illustrated in List 1 using BNF notation. It is composed of a list of query (or condition) and response pairs. Topic is the primary component that represents a query-response pair in the grammar.

Scripts are interpreted and loaded into memory by script interpreter when the agent start running. The condition part in each topic is transformed into a Boolean expression and the keywords listed in the condition part are transformed into a regular expression as in Fig. 3.

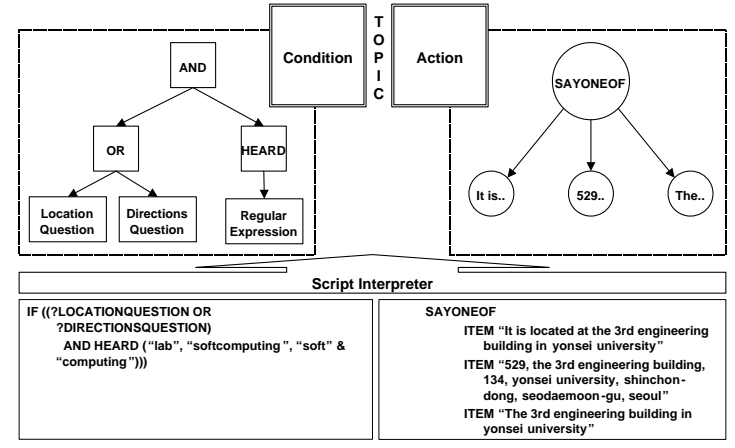

Fig. 3. Internal representation of a part of the script

the condition part of each topic is compared with the goal information, the query, and the keyword list extracted during the goal inference. This process returns the scores of all the topics as a result. Different types of matching components, like goals, keywords, or Boolean operators, are assigned different scores depending on their types. When all the topics are assigned scores, the highest topic is presented to the user as a response to his query.

\section{Simulation Results}

The conversational agent has been applied to the introduction of a website to show the usability and possibility. Although it is necessary to assign initial probabilities from the 
analysis of the corpus in case of serious real-world problems, they are given such that they represent the frequencies of the occurrence of the evidence variables in goals because it is sufficient for this tiny model in demonstrating the possibility of the modeling method. Following is an actual conversation with a user.

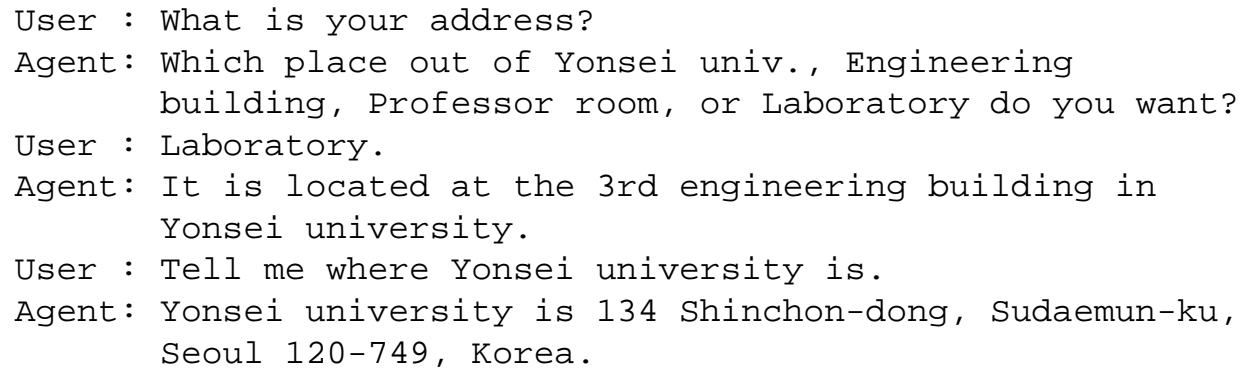

When a user asks the agent "What is your address?," the agent searches synonyms to be replaced with their representative words. In this case, no words are replaced. Then the agent compares some words of the query with the variables of the Bayesian network. As this query contains 'what' and 'address', which are evidences of 'Location', 'Web field', and 'Web intention', their probabilities are looked up from the predefined conditional probability tables as in Fig. 4 (a). Since the probability of 'Location' is over

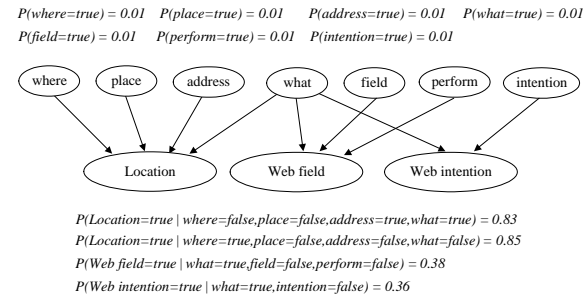

(a) 'Location'

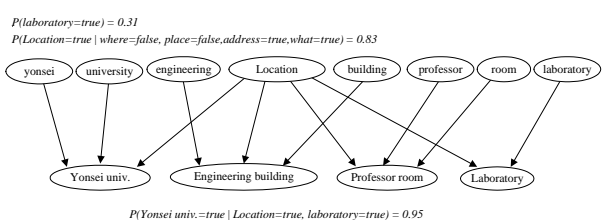

(b) 'Laboratory selection'

Fig. 4. Goal searching procedures

the threshold probability 0.8 and greater than the other goals, the agent infers that the user's goal is 'Location'. At this moment, the agent needs more information which of the four detailed goals the user actually wants to know as in fig. 4 (b). The agent asks the user "Which place out of Yonsei univ., Engineering building, Professor room, or Laboratory?" The answer, "Laboratory," makes the 'Laboratory' variable true and thus the agent infers that user goal is 'Laboratory'. With this inferred user goal, the script is searched producing the response "It is located at the 3rd building in Yonsei university."

When the user asks the agent "Tell me where Yonsei university is," the agent takes the 'where' word which is an evidence variable of the Bayesian network. P(Location $\mid$ where) 


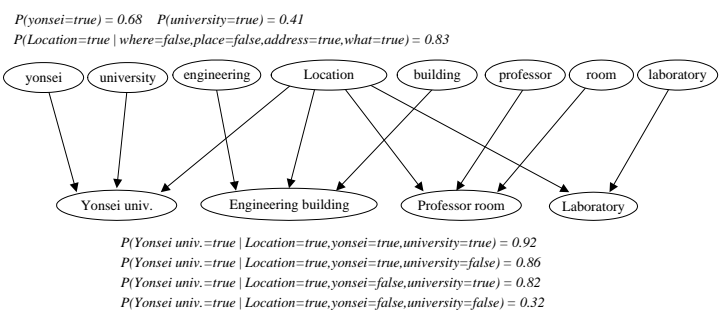

Fig. 5. Inferring detailed user goals

is 0.85 as shown in Fig. 4 (a), because 'Location' is conditionally independent given 'where'. After inferring the user's goal, the agent gathers more information for the next sub goal. The agent takes 'yonsei' and 'university' words in the query and infers 'Yonsei univ.' with the words as in fig. 5. After inferring 'Yonsei univ.', the agent finally answers to the user "Yonsei university is 134 Shinchon-dong, Sudaemun-ku, Seoul 120-749, Korea."

\section{Conclusion}

In this paper, we have described a conversational agent that can give responses more consistent with user's goals in a specific domain. A Bayesian network is used to construct the user model in a specific domain. Although we have constructed the user model of limited goals in a specific domain, the results show that this has the possibility of interacting with users more consistently with their goals. As a further research, we plan to construct more general user models that are independent on domains with Bayesian network.

\section{References}

1. J. Weizenbaun: ELIZA - a Computer Program for the Study of Natural Language Communication between Man and Machine. Communications of the ACM 9(1). (1965) 36-45

2. D. Pynadath and M. Wellman: Accounting for Context in Plan Recognition with Application to Traffic Monitoring. Proc. of the Eleventh Conf. on Uncertainty in Artificial Intelligence. (1995) 472-481

3. D. Albrecht et al: Towards a Bayesian Model for Keyhole Plan Recognition in Large Domains. Proc. of the Sixth Int. Conf. on User Modeling. (1997) 365-376.

4. E. Horvitz and T. Paek: A Computational Architecture for Conversation. Proc. of the Seventh Int. Conf. on User Modeling. (1999) 201-210

5. F.V. Jensen: An Introduction to Bayesian Networks, Springer-Verlag. (1996)

6. N. Friedman et al: Using Bayesian Networks to Analyze Expression Data. Proc. of the Fourth Annual Int. Conf. on Computational Molecular Biology. (2000) 127-135

7. J.F. Allen: Mixed-Initiative Interaction. IEEE Intelligent Systems 5 (1999) 14-16 